\title{
Dementia care and COVID-19 pandemic: a necessary digital revolution
}

\author{
Luca Cuffaro $^{1}$ - Francesco Di Lorenzo ${ }^{2}$. Simona Bonavita ${ }^{3} \cdot$ Gioacchino Tedeschi $^{3}$ - Letizia Leocani ${ }^{4,5}$ (D) \\ Luigi Lavorgna ${ }^{3}$
}

Received: 6 May 2020 / Accepted: 30 May 2020 / Published online: 17 June 2020

(C) Fondazione Società Italiana di Neurologia 2020

\begin{abstract}
Due to the COVID-19 pandemic, most memory clinics have had to suspend their activities. On the other hand, international dementia experts have recommended to provide urgently worldwide support for people living with dementia. This situation urges to play out new strategies to guarantee adequate care. Telemedicine and digital technology (DT) devices, such as smartphones, can be very helpful in remote monitoring and care. Technological devices such as videoconference or smartphone apps might be used for follow-up visits and support to patients and caregivers and to acquire digital markers of clinical progression. Hopefully, this dramatic situation would facilitate the process of progressive familiarization of neurologists with telemedicine and DT approach.
\end{abstract}

Keywords Dementia $\cdot$ Alzheimer's disease $\cdot$ Digital technology $\cdot$ Telemedicine $\cdot$ Teleneurology $\cdot$ COVID

The severe acute respiratory syndrome coronavirus 2 (SARSCoV-2) rapidly caused a pandemic of coronavirus disease 2019 (COVID-19), greatly stressing the healthcare systems worldwide. The social restrictions have had a substantial impact on the management of outpatient clinics with cancelations or postponement of outpatient visits [1]. Vulnerable patients, as elderly people with cognitive impairment (CI)

Luca Cuffaro and Francesco Di Lorenzo contributed equally to this work.

On behalf of the Digital Technologies Web and Social Media Study Group of the Italian Society of Neurology, Italy.

Letizia Leocani

leocani.letizia@hsr.it

1 Department of Biomedicine, Neuroscience and Advanced Diagnostics, University of Palermo, Palermo, Italy

2 Non-Invasive Brain Stimulation Unit, IRCCS Fondazione Santa Lucia, Rome, Italy

3 Department of Medical, Surgical, Neurological, Metabolic and Aging Sciences, II Clinic of Neurology, University of Campania "Luigi Vanvitelli", Naples, Italy

4 Neurorehabilitation Unit, Experimental Neurophysiology Unit Institute of Experimental Neurology-INSPE, IRCCS San Raffaele Hospital, Milan, Italy

5 University Vita-Salute San Raffaele, Milan, Italy or dementia, have an increased risk of serious morbidity, admission to intensive care units, and death when infected with COVID-19 because of their advanced age and frequent medical comorbidities. Consequently, they have been highly recommended to adhere to social distancing, increasing caregiver burden. Furthermore, these measures might lead patients with $\mathrm{CI}$ to a faster cognitive deterioration and to worsening of behavioral and psychological symptoms of dementia, inducing in turn higher caregiver distress. National and International Societies have made several recommendations about dementia care addressed to all dementia patients and their caregivers [2].

Unlike other neurological disorders, people with CI do not usually require frequent visits; however, as the general population age increases, memory clinics tend to be increasingly overcrowded due to diagnostic processes of early stage CI patients (requiring closer monitoring of symptoms progression) and long-term management of chronic patients.

As future COVID-19 outbreaks cannot be excluded to occur in the upcoming months, neurologists are forced to adopt new strategies to guarantee minimal standards of care for their patients and limit hospital access of elderly people to protect them from infection.

COVID-19 pandemic is accelerating telemedicine and digital technology (DT) use for chronic neurological diseases management [3] 
Recently, increasing research investigated the feasibility and benefits of DTs to remotely monitor people with dementia.

The American Academy of Neurology has developed a guidance for clinicians and practices to implement telemedicine services amid the COVID-19 crisis, with obvious limitations to some specific parts of the neurological examination (e.g., fundoscopy, reflexes, etc.) which, nonetheless, may be not fundamental, particularly in the follow-up interview of a patient already diagnosed with CI [4].

As postponing planned referrals will increase the future workload and delay proper update in the management of people with CI, a follow-up visit with telemedicine approach may provide timely referrals, thus avoiding delays or engulfment of the waiting lists. Specifically, videoconferences and video monitoring have been shown to be as efficient as in-person visits in evaluation of daily living activities and global cognition [5].

Also, the diagnostic process can be facilitated through telemedicine and DT. Indeed, although instrumental examinations are paramount for the detection of physiopathological biomarkers of disease (e.g., cerebrospinal fluid, amyloid PET), a preliminary telemedicine consultation could be helpful in individuating the patients who actually need to be further tested.

Accurate neuropsychological evaluations are possible through telemedicine. Although further studies are needed to test the reliability of complex tasks requiring motor and visual abilities, there is growing scientific evidence supporting the use of videoconferencing technology in the remote administration of neuropsychological tests, particularly those that rely on verbal responses [6].

There is preliminary evidence that smartphone-based assessment of episodic memory [7], cognitive processing speed [8], and visual attention [9] are reliable tools to evaluate cognitive functions in preclinical populations and to monitor progression of cognitive decline in patients with CI.

Telemedicine and e-health services are also available to support care for older adults and family caregivers. Internet-based technology can support people at early stages of CI through electronic reminders, daily activities, and cognitive stimulation games [10]. Even more important, most of the new DTs are devoted to support caregivers. Readily available web-based training and psycho-educational programs [11] improve knowledge and competence for caregiving, reducing caregiver psychological burden [12]. Also, mobile text messaging, video-recording, and chat forums can be useful for meeting specific caregivers' needs. Crucially, there is an increasing request and high level of satisfaction for technological support in assisting caregivers [13].

Thus, the activity of a memory clinic may partially continue during the current pandemic, albeit with some expedients. The adoption of a virtual clinic is necessary for avoiding traditional face-to-face visits for vulnerable patients, without hampering the quality of neurological consultation. We recommend the use of electronic health record systems (that could be easily implemented through online tools, such as Google Forms) and e-mails for faster communication (e.g., report or visualization of diagnostic examinations and medical prescriptions). Then, after the first contact with patients and caregivers, we suggest tailoring the best tele-medical support. Neuropsychologists should be trained also to familiarize with video conference testing, and some tests should be readily converted into a digital version. We believe that it is necessary to make a preliminary selection of patients based on their diagnostic/management process in order to avoid management or diagnostic delays. Depending on patients and caregiver willingness, a teleneurology consultation could be conducted via video call to obtain more details on patient's condition, and this is strongly advised in case of new referrals.

The teleneurology may offer a cost-effective and practical method for delivering interventions to dementia patients and caregivers, especially through the COVID19 pandemic, favoring the adoption of new ways to deliver medical care.

However, this modern approach could encounter several limitations, due to the availability of appropriate conditions to perform tele-consultations (e.g., quality of connection, patient's ownership of webcam), knowledge and familiarity of patients and caregivers with these modern technologies, and ethical concerns which require caution and specific privacy policy to protect sensitive data such as compliance to the national General Data Protection Regulation.

Independently from the current COVID 19 pandemic, the application of technological devices in dementia care may lead to several advantages such as improving access and quality of care, servicing patients coming from rural communities, and supporting caregivers. The next step is to encourage a widespread adoption of telemedicine services promoted by healthcare boards directives.

Here we suggested different telemedicine and DT approaches that can be easily used to keep on providing acceptable standards of care during this pandemic. Once the needs of people with $\mathrm{CI}$ and their caregivers will be identified, and the strategies for their assistance established, an appropriate technical support could ease the life of many people [13]. Follow-up visits, cognitive and digital markers, and effective supporting measurements can be very helpful at the present time.

Acknowledgments Digital Technologies, Web and Social Media Study Group of Italian Neurological Society (SIN). Giovanni Mancardi, Alessandro Padovani, Francesco Brigo, Marinella Clerico, Eleonora Cocco, Luca Prosperini, Maria Laura Stromillo, Marcello Moccia, Roberta Lanzillo, Vincenzo Brescia Morra, Antonio Russo, Bruno Giometto, Anna Maria Repice, Gianmarco Abbadessa, Alberto Lerario, Francesca Trojsi, Rosa Iodice, Sebastiano Bucello, Pietro Annovazzi, Antonio De Martino, Alessandro Bombaci, Francesco Iodice, Michele Romoli, Giuseppina Miele, Giulia Straccia, Marcello Silvestro. 


\section{Compliance with ethical standards}

Conflict of interest Authors report no disclosures relevant to the manuscript.

Ethical approval None.

\section{References}

1. Bonavita S, Tedeschi G, Atreja A, Lavorgna L (2020) Digital triage for people with multiple sclerosis in the age of COVID-19 pandemic. Neurol Sci 41(5):1007-1009. https://doi.org/10.1007/s10072020-04391-9

2. Coronavirus (COVID-19): Tips for Dementia Caregivers [online]. Alzheimers Dis. Dement. Accessed at: https://alz.org/help-support/ caregiving/coronavirus-(covid-19)-tips-for-dementia-care. Accessed April 24, 2020

3. Caso V, Federico A (2020) No lockdown for neurological diseases during COVID19 pandemic infection. Neurol Sci 41(5):999-1001. https://doi.org/10.1007/s10072-020-04389-3

4. Hatcher-Martin JM, Adams JL, Anderson ER, Bove R, Burrus TM, Chehrenama M, Dolan O'Brien M, Eliashiv DS, Erten-Lyons D, Giesser BS, Moo LR, Narayanaswami P, Rossi MA, Soni M, Tariq N, Tsao JW, Vargas BB, Vota SA, Wessels SR, Planalp H, Govindarajan R (2020) Telemedicine in neurology: telemedicine work Group of the American Academy of neurology update. Neurology 94:30-38

5. Kim H, Jhoo JH, Jang J-W (2017) The effect of telemedicine on cognitive decline in patients with dementia. J Telemed Telecare 23: 149-154

6. Brearly TW, Shura RD, Martindale SL, Lazowski RA, Luxton DD, Shenal BV, Rowland JA (2017) Neuropsychological test administration by videoconference: a systematic review and metaanalysis. Neuropsychol Rev 27:174-186

7. Lancaster C, Koychev I, Blane J, Chinner A, Wolters L, Hinds C (2020) Evaluating the feasibility of frequent cognitive assessment using the Mezurio smartphone app: observational and interview study in adults with elevated dementia risk. JMIR MHealth UHealth 8:e16142

8. Brouillette RM, Foil H, Fontenot S, Correro A, Allen R, Martin CK, Bruce-Keller AJ, Keller JN (2013) Feasibility, reliability, and validity of a smartphone based application for the assessment of cognitive function in the elderly. PLoS One 8:e65925

9. Tieges Z, Stíobhairt A, Scott K, Suchorab K, Weir A, Parks S, Shenkin S, MacLullich A (2015) Development of a smartphone application for the objective detection of attentional deficits in delirium. Int Psychogeriatr 27:1251-1262

10. Bossen A, Kim H, Steinhoff A, Strieker M, Williams K (2015) Emerging roles for telemedicine and smart technologies in dementia care. Smart Homecare Technol TeleHealth 3:49-57

11. Godwin KM, Mills WL, Anderson JA, Kunik ME (2013) Technology-driven interventions for caregivers of persons with dementia: a systematic review. Am J Alzheimers Dis Other Dement 28:216-222

12. Finkel S, Czaja SJ, Schulz R, Martinovich Z, Harris C, Pezzuto D (2007) E-care: a telecommunications technology intervention for family caregivers of dementia patients. Am J Geriatr Psychiatry Off J Am Assoc Geriatr Psychiatry 15:443-448

13. Armstrong N, Nugent C, Moore G, Finlay D (2010) Using smartphones to address the needs of persons with Alzheimer's disease. Ann Telecommun -Annales des Télécommunications 65(910):485-495. https://doi.org/10.1007/s12243-010-0165-3

Publisher's note Springer Nature remains neutral with regard to jurisdictional claims in published maps and institutional affiliations. 